\title{
On Optimal Throughput-Energy Curve for Multi-hop Wireless Networks
}

\author{
Canming Jiang Yi Shi Y. Thomas Hou \\ The Bradley Department of Electrical and Computer Engineering \\ Virginia Polytechnic Institute and State University \\ Blacksburg, VA, USA
}

\author{
Sastry Kompella \\ Information Technology Division \\ U.S. Naval Research Laboratory \\ Washington, DC, USA
}

\begin{abstract}
Network throughput and energy consumption are two important performance metrics for a multi-hop wireless network. Current state-of-the-art is limited to either maximizing throughput under some energy constraint or minimizing energy consumption while satisfying some throughput requirement. In this paper, we take a multicriteria optimization approach to offer a systematic study on the relationship between the two performance objectives. We show that the solution to the multicriteria optimization problem is equivalent to finding an optimal throughput-energy curve, which characterizes the envelope of the entire throughput-energy region. We prove some important properties of the optimal throughput-energy curve. For case study, we consider both linear and nonlinear throughput functions. In the linear case, we characterize the optimal throughput-energy curve precisely through parametric analysis, while in the nonlinear case, we use a piece-wise linear approximation to approximate the optimal throughput-energy curve with arbitrary accuracy. Our results offer important insights on exploiting the trade-off between the two performance metrics.
\end{abstract}

\section{INTRODUCTION}

Since the inception of multi-hop wireless networks, throughput and energy consumption are two key performance metrics that bear in the minds of network designers and operators. Throughput is clearly the first and foremost performance consideration, as users of a multi-hop wireless network increasingly wish such network can offer comparable experience as its counterpart wired networks. On the other hand, energy consumption is also regarded as a key performance consideration, as many types of multi-hop wireless networks (e.g., ad hoc network, sensor network) are battery-powered and are constrained with energy at each node.

To date, there is a vast amount of literature on these two performance metrics. For network throughput, people have been trying to maximize it either at different layers (e.g., throughput-efficient scheduling algorithms [8], [26], [36], [38], throughput-efficient routing algorithms [6], [13], [32]) or jointly across multiple layers (e.g., [1], [2], [7], [12], [24], [27]). For energy, people are trying to conserve/minimize its consumption while meeting certain service requirements (e.g., energy-efficient scheduling and MAC schemes [17], [21], [34], [35], [37], [39], energy-efficient routing protocol [15], [18], [23]).

We have also witnessed quite some studies exploring the interaction between network throughput and energy consumption in the context of either maximizing network throughput under energy (or power) constraints (e.g., [9], [14], [31]) or minimizing energy consumption while satisfying some throughput constraints (e.g., [5], [10], [24], [25], [31]). Although many of these prior efforts were able to offer some optimal solutions, none of them can offer a holistic view on how the maximum network throughput changes as a function of network energy consumption, i.e., the so-called optimal throughput-energy curve in this paper.

The significance of optimal throughput-energy curve is three-fold. First, this shows the envelop of the entire throughput-energy region, which offers a global perspective on the throughput-energy tradeoff. In contrast, a solution to traditional problems such as maximizing throughput under energy constraints or minimizing energy under throughput constraints only represents a single point on this curve. Second, each time when the requirement on either network throughput or energy consumption changes, one can use the optimal throughput-energy curve to find a new optimal tradeoff between throughput and energy immediately, rather than resorting to solving a new optimization problem. Finally, the optimal throughput-energy curve shows us the existence of a saturation point, beyond which the throughput can no longer be further increased, regardless of how much additional energy is used.

In this paper, we conduct a systematic study on the optimal relationship between network throughput and energy consumption for a multi-hop wireless network. We tackle this problem through a multicriteria optimization formulation, i.e., maximizing network throughput while minimizing total power in the network. Our main contributions can be summarized as follows.

- Through multicriteria optimization formulation, we find that the optimal solutions of this multicriteria optimization problem can be found by obtaining the so-called optimal throughput-energy curve.

- We find a number of important properties associated with the optimal throughput-energy curve, such as nondecreasing, concave, the existence of a saturation point, and strictly increasing between zero and the saturation point.

- For case study, we consider two cases where the throughput functions are linear and nonlinear, respectively. 
- In the linear case, we show that the optimal throughput-energy curve can be characterized precisely via parametric analysis.

- In the nonlinear case, we show that the optimal throughput-energy curve can be approximated by piece-wise linear segments with arbitrary desired accuracy.

The remainder of this paper is organized as follows. In Section II, we describe our network model. In Section III, we present a multicriteria formulation that maximizes network throughout while minimizing energy consumption in a multihop wireless network. We show that finding the optimal solution to this multicriteria optimization problem is equivalent to finding the optimal throughput-energy curve. We also present some important properties associated with the optimal throughput-energy curve. Section IV and Section V present two case studies when the throughput functions are linear and nonlinear, respectively. Section VI concludes this paper.

\section{NETWORK MODEL}

We consider a general multi-hop wireless network with a set of $\mathcal{N}$ nodes. A potential directed link $(i, j), i, j \in \mathcal{N}$, exists between two nodes $i$ and $j$ if and only if the distance between $i$ and $j$ is smaller than a transmission range. Denote $\mathcal{L}$ the set of potential links in the network. To focus on throughput and energy performance, we simplify link layer scheduling by employing orthogonal channels among the links, similar to that in [12], [22], [29].

Denote $\mathcal{M}$ a set of user (unicast) communication sessions in the network. Denote $\operatorname{src}(m)$ and $\operatorname{dst}(m)$ the source and destination nodes of session $m \in \mathcal{M}$, respectively. Denote $r(m)$ the rate of session $m \in \mathcal{M}$. We consider a general flow routing strategy where flow splitting (i.e., multi-path) is allowed. On link $l$, denote $r_{l}(m)$ the data rate that is attributed to session $m \in \mathcal{M}$. Denote $\mathcal{L}_{i}^{\text {Out }}$ and $\mathcal{L}_{i}^{\text {In }}$ the sets of potential outgoing and incoming links at node $i$, respectively. Then we have the following flow balance equations for multi-hop routing.

- If node $i$ is the source node of session $m$, i.e., $i=\operatorname{src}(m)$, then

$$
\sum_{l \in \mathcal{L}_{i}^{\text {Out }}} r_{l}(m)=r(m)
$$

- If node $i$ is an intermediate relay node along the path of session $m$, i.e., $i \neq \operatorname{src}(m)$ and $i \neq \operatorname{dst}(m)$, then

$$
\sum_{l \in \mathcal{L}_{i}^{\text {Out }}}^{l \neq(i, \operatorname{src}(m))} r_{l}(m)=\sum_{l \in \mathcal{L}_{i}^{\text {In }}}^{l \neq(\operatorname{dst}(m), i)} r_{l}(m) .
$$

- If node $i$ is the destination node of session $m$, i.e., $i=$ $\operatorname{dst}(m)$, then

$$
\sum_{l \in \mathcal{L}_{i}^{\text {In }}} r_{l}(m)=r(m) .
$$

It can be easily verified that once (1) and (2) are satisfied, then (3) is also satisfied. As a result, it is sufficient to list only (1) and $(2)$ in a formulation.
For power control at each node, we employ a simple "on/off" control, which has been used for energy-saving in wireless networks (see e.g., [28], [30]). When a link is "on", the transmitter of this link transmits at a fixed power level $P_{T}$; when the link is "off" (for energy conservation), the transmitter of this link does not expend any power for transmission. To quantify the percentage of time that the link is in different state, denote $\alpha_{l}\left(0 \leq \alpha_{l} \leq 1, l \in \mathcal{L}\right)$ the fraction of time within a time frame that link $l$ is "on".

Based on this on/off energy conservation model, the average rate of link $l$ can be computed as

$$
C_{l}=\alpha_{l} \cdot B \log _{2}\left(1+\frac{P_{T} \cdot g_{l}}{\eta B}\right),
$$

where $B$ is the channel bandwidth, $g_{l}$ is channel gain between the transmitter and receiver of link $l$ and $\eta$ is the ambient Gaussian noise density. Note the absence of interference in (4), due to our use of orthogonal channels in the network.

On link $l$, we have the following flow rate constraint:

$$
\sum_{m \in \mathcal{M}} r_{l}(m) \leq C_{l}, \text { for all } l \in \mathcal{L},
$$

which states that the aggregate flow rates from all sessions traversing link $l$ cannot exceed the achievable rate of this link.

\section{Throughrut-Energy Curve And Its Properties}

\section{A. Multicriteria Formulation}

In this paper, we are interested in a multicriteria optimization problem, i.e., how to maximize network throughput while minimizing energy consumption at the same time. We now give a formulation of this problem.

Denote $h(\cdot)$ as a concave and nondecreasing utility function. We define the network throughput utility $U$ as follows:

$$
U=\sum_{m \in \mathcal{M}} h[r(m)]
$$

where $r(m)$ is the rate of session $m \in \mathcal{M}$. Note that in the special case when $h[r(m)]=r(m)$, then $U$ is simply the sum of throughput in the network; in the case when $h[r(m)]=$ $\ln [r(m)], U$ is called proportional fairness [20].

Now we consider energy consumption. Note that when a link is active, the rate of energy consumption includes energy consumption both for transmission and reception. Then the network energy consumption rate $P$ in the network can be defined as follows:

$$
P=\sum_{l \in \mathcal{L}} \alpha_{l} \cdot\left(P_{T}+P_{R}\right)
$$

where $\alpha_{l}$ is the fraction of time within a time frame that link $l$ is active, $P_{T}$ is the transmission power, and $P_{R}$ is the reception power. 
With the above two definitions, our multicriteria optimization problem can be formulated as follows.

$$
\begin{aligned}
\text { MOPT } \min & P=\sum_{l \in \mathcal{L}} \alpha_{l} \cdot\left(P_{T}+P_{R}\right) \\
\max & U=\sum_{m \in \mathcal{M}} h[r(m)] \\
\text { s.t. } & \text { Constraints }(1),(2),(4) \text { and }(5) \\
& r(m), r_{l}(m) \geq 0,0 \leq \alpha_{l} \leq 1 .
\end{aligned}
$$

Note that the two objective functions, $P$ and $U$, are conflicting objectives. For example, when $P$ is minimized (i.e., 0 ), $U$ is also 0 and is not maximized. So there does not appear to exist an optimal solution in our problem that optimizes both objectives simultaneously.

Given that an optimal solution does not exist, a natural question to ask is what kind of solutions should we pursue when investigating problem MOPT? Before answering this question, we first clarify how to compare two feasible solutions. Denote $\left(P_{1}, U_{1}\right)$ and $\left(P_{2}, U_{2}\right)$ the objective pairs of two different feasible solutions $\mathbf{x}_{1}$ and $\mathbf{x}_{2}$, respectively. We say objective pair $\left(P_{1}, U_{1}\right)$ dominates $\left(P_{2}, U_{2}\right)$ if $P_{1} \leq P_{2}$ and $U_{1} \geq U_{2}$. This means that solution $\mathbf{x}_{1}$ uses no more energy than solution $\mathbf{x}_{2}$ to achieve the same or more throughput, i.e., $\mathbf{x}_{1}$ is better than $\mathbf{x}_{2}$. With this clarification, it is clear that our goal should be to find solutions that are not dominated by any other solutions. That is, we want to find solutions with their objective pair $\left(P^{\dagger}, U^{\dagger}\right)$ such that there does not exist another solution with objective pair $(P, U)$ such that $P \leq P^{\dagger}$ and $U \geq U^{\dagger}$. Such solutions are called Pareto optimal solutions (also called efficient solutions in [16]) and the objective value pair $\left(P^{\dagger}, U^{\dagger}\right)$ corresponding to a Pareto optimal solution is called a Pareto optimal point. Pareto optimal solutions are those for which improvement in one objective can only be achieved with the deterioration of the other objective.

For our problem, we find that it is difficult to obtain all Pareto optimal solutions directly. Instead, we can find a solution $\mathbf{x}^{*}$ with its objective pair $\left(P^{*}, U^{*}\right)$ such that there does not exist another solution $\mathbf{x}$ with its objective pair $(P, U)$ satisfying $P<P^{*}$ and $U>U^{*}$. That is, there does not exist a solution $\mathbf{x}$ that can use less energy than solution $\mathbf{x}^{*}$ to achieve more throughput. Such solutions are called weakly Pareto optimal solutions (also called weakly efficient solutions in [16]) and the objective value pair $\left(P^{*}, U^{*}\right)$ corresponding to such a solution is called a weakly Pareto optimal point. Note that Pareto optimal points are also weakly Pareto optimal, but weakly Pareto optimal points are not always Pareto optimal. Weakly Pareto optimal solutions are those for which improvement in both objectives simultaneously is impossible, but improvement on one objective without deteriorating the other is possible. Once we find all the weakly Pareto optimal solutions, we can identify a subset of solutions that are Pareto optimal based on its definition.

\section{B. Throughput-Energy Curve}

Instead of solving MOPT directly, let's consider a simpler single objective optimization problem for a given $P$ (i.e., fixing one of the objective value). That is,

$$
\begin{aligned}
\operatorname{OPT}(P) \quad \max & \sum_{m \in \mathcal{M}} h[r(m)] \\
\text { s.t. } & \sum_{l \in \mathcal{L}} \alpha_{l}\left(P_{T}+P_{R}\right)=P \\
& \text { All constraints in MOPT } \\
& r(m), r_{l}(m) \geq 0,0 \leq \alpha_{l} \leq 1 .
\end{aligned}
$$

We now show that the optimal solution to $\mathrm{OPT}(P)$ is a weakly Pareto optimal solution to MOPT.

Lemma 1: Let $\mathbf{x}^{*}=\left\{r^{*}(m), r_{l}^{*}(m), \alpha_{l}^{*} \mid l \in \mathcal{L}, m \in \mathcal{M}\right\}$ be an optimal solution to OPT $(P)$ for a given value of $P^{*}$ with a corresponding objective value $U^{*}$, then $\mathrm{x}^{*}$ is a weakly Pareto optimal solution to MOPT.

Lemma 1 can be proved by contradiction. Due to page limitation, we refer readers to [19].

Denote the range of $P$ to be $\left[0, P_{\max }\right]$, where $P_{\max }$ can be obtained by setting $\alpha_{l}=1$ for all $l \in \mathcal{L}$. That is, $P_{\max }=$ $\sum_{l \in \mathcal{L}}\left(P_{T}+P_{R}\right)=|\mathcal{L}| \cdot\left(P_{T}+P_{R}\right)$. If one can enumerate all possible $P \in\left[0, P_{\max }\right]$ and obtain their corresponding optimal solutions via OPT $(P)$, then based on Lemma 1, all these solutions are weakly Pareto optimal solutions.

Now we show the converse is also true, i.e., any weakly Pareto optimal point $(P, U)$ of MOPT can be obtained by a corresponding problem of OPT $(P)$.

Lemma 2: Each weakly Pareto optimal point $(P, U)$ of $M O P T$ can be obtained by solving an instance of $O P T(P)$.

The proof of Lemma 2 is based on contradiction. Due to page length, we refer readers to [19].

Based on Lemmas 1 and 2, we conclude that each weakly Pareto optimal point $(P, U)$ of MOPT uniquely corresponds to the same $(P, U)$ generated by an optimal solution of OPT $(P)$. Thus, by finding the optimal $U$ for each OPT $(P)$, $P \in\left[0, P_{\text {max }}\right]$, we can obtain all the weakly Pareto optimal points of MOPT. This gives us a mapping from $P$ to $U$, which we denote as $f: P \rightarrow U$. Intuitively, this says that for any weakly Pareto optimal point $(P, U), U=f(P)$ is the maximum throughput utility that the network can deliver. We call $U=f(P)$ the optimal throughput-energy curve, which we formally define as follows.

Definition 1: (Optimal Throughput-Energy Curve) For all $P \in\left[0, P_{\max }\right]$, the mapping $f: P \rightarrow U$ via solving $O P T(P)$ constitutes an optimal throughput-energy curve $U=f(P)$.

\section{Properties}

In this section, we present several interesting properties for the optimal throughput-energy curve. These properties are important for us to understand the general behavior of this curve and to characterize this curve under specific throughput utility function.

Property 1: $U=f(P)$ is a nondecreasing function over $0 \leq P \leq P_{\max }$. 
This property is easy to understand intuitively. It says that the throughput will not decrease when energy is increased. The proof is quite straightforward and is omitted here.

Property 2: $U=f(P)$ is a concave function.

Proof: Based on the definition of a concave function, we need to prove that for any $P_{1}, P_{2}$, and $\lambda\left(0 \leq P_{1} \leq P_{\max }\right.$, $0 \leq P_{2} \leq P_{\max }$, and $\left.0 \leq \lambda \leq 1\right)$, we have $f\left[\lambda P_{1}+(1-\right.$ d) $\left.P_{2}\right] \geq \lambda f\left(P_{1}\right)+(1-\lambda) f\left(P_{2}\right)$.

Suppose the optimal solution of $\operatorname{OPT}\left(P_{1}\right)$ is $\mathbf{x}^{\mathbf{1}}=$ $\left\{r^{1}(m), r_{l}^{1}(m), \alpha_{l}^{1} \mid l \in \mathcal{L}, m \in \mathcal{M}\right\}$ with the optimal objective value $f\left(P_{1}\right)$. Suppose the optimal solution of $\operatorname{OPT}\left(P_{2}\right)$ is $\mathbf{x}^{\mathbf{2}}=\left\{r^{2}(m), r_{l}^{2}(m), \alpha_{l}^{2} \mid l \in \mathcal{L}, m \in \mathcal{M}\right\}$ with the optimal objective value $f\left(P_{2}\right)$. We will construct a feasible solution for $\operatorname{OPT}\left(\lambda P_{1}+(1-\lambda) P_{2}\right)$ with an objective value being at least $\lambda f\left(P_{1}\right)+(1-\lambda) f\left(P_{2}\right)$. Then, for the optimal solution of $\mathrm{OPT}\left(\lambda P_{1}+(1-\lambda) P_{2}\right)$, its optimal objective value $f\left(\lambda P_{1}+(1-\lambda) P_{2}\right)$ is at least $\lambda f\left(P_{1}\right)+(1-\lambda) f\left(P_{2}\right)$.

The constructed solution is $\lambda \cdot \mathbf{x}^{1}+(1-\lambda) \cdot \mathbf{x}^{2}$. We now show that it is a feasible solution to $\operatorname{OPT}\left(\lambda P_{1}+(1-\lambda) P_{2}\right)$. First, we verify that $\lambda \cdot \mathbf{x}^{1}+(1-\lambda) \cdot \mathbf{x}^{2}$ satisfies constraint (6). We have $\sum_{l \in \mathcal{L}}\left[\lambda \alpha_{l}^{1}+(1-\lambda) \alpha_{l}^{2}\right]\left(P_{T}+P_{R}\right)=\lambda \sum_{l \in \mathcal{L}} \alpha_{l}^{1}\left(P_{T}+\right.$ $\left.P_{R}\right)+(1-\lambda) \sum_{l \in \mathcal{L}} \alpha_{l}^{2}\left(P_{T}+P_{R}\right)=\lambda P_{1}+(1-\lambda) P_{2}$, i.e., constraint (6) holds. Second, we note that the region defined by constraints (1), (2), (4) and (5) is a convex region. Since $\mathbf{x}^{1}$ and $\mathbf{x}^{2}$ are both in this region, we have that $\lambda \cdot \mathbf{x}^{1}+(1-$ $\lambda) \cdot \mathbf{x}^{2}$ is also in this region, i.e., $\lambda \cdot \mathbf{x}^{1}+(1-\lambda) \cdot \mathbf{x}^{2}$ satisfies constraints (1), (2), (4) and (5). Therefore, the constructed solution satisfies all constraints in $\operatorname{OPT}\left(\lambda P_{1}+(1-\lambda) P_{2}\right)$ and thus is feasible.

Now we calculate the objective value achieved by solution $\lambda \cdot \mathbf{x}^{1}+(1-\lambda) \cdot \mathbf{x}^{\mathbf{2}}$, which is

$$
\begin{aligned}
& \sum_{m \in \mathcal{M}} h\left[\lambda r^{1}(m)+(1-\lambda) r^{2}(m)\right] \\
\geq & \sum_{m \in \mathcal{M}}\left\{\lambda h\left[r^{1}(m)\right]+(1-\lambda) h\left[r^{2}(m)\right]\right\} \\
= & \lambda \sum_{m \in \mathcal{M}} h\left[r^{1}(m)\right]+(1-\lambda) \sum_{m \in \mathcal{M}} h\left[r^{2}(m)\right] \\
= & \lambda f\left(P_{1}\right)+(1-\lambda) f\left(P_{2}\right),
\end{aligned}
$$

where the first inequality holds since $h(\cdot)$ is a concave function. Therefore, we have constructed a feasible solution to $\operatorname{OPT}\left(\lambda P_{1}+(1-\lambda) P_{2}\right)$ with its objective value greater than or equal to $\lambda f\left(P_{1}\right)+(1-\lambda) f\left(P_{2}\right)$. This completes the proof.

The next two properties further spell out the particular shape of the concave throughput-energy curve.

Property 3: There exists a saturation point $\left(P_{s}, U_{s}\right)$ on the optimal throughput-energy curve $f(P)$ such that $f(P)=U_{s}$, for $P \in\left[P_{s}, P_{\text {max }}\right]$ and $f(P)<U_{s}$ for $P<P_{s}$.

The above property says that the last segment of the optimal throughput-energy curve is flat after the saturation point (see Fig. 1). Now, we show how to compute the saturation point $\left(P_{s}, U_{s}\right)$ as follows. We first compute the maximum achievable network throughput $U_{s}$ under OPT $\left(P_{\max }\right)$. Once we have $U_{s}$, we can find the minimum network energy consumption rate $P_{s}$

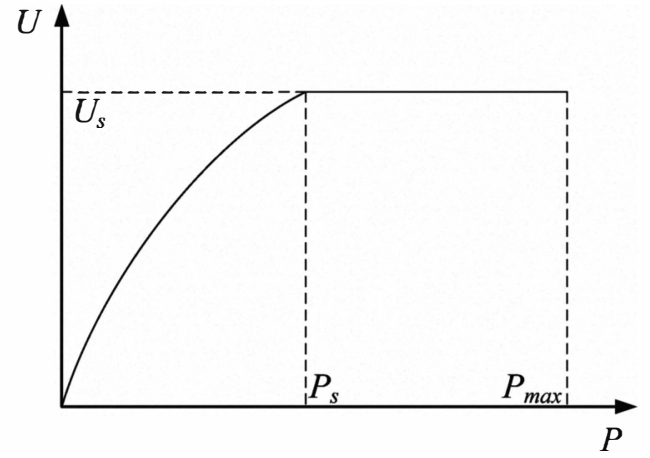

Fig. 1. The shape of an optimal throughput-energy curve.

that can achieve this $U_{s}$ by solving the following optimization problem:

$$
\begin{aligned}
P_{s}=\min & \sum_{l \in \mathcal{L}} \alpha_{l} \cdot\left(P_{T}+P_{R}\right) \\
\text { s.t. } & \sum_{m \in \mathcal{M}} h[r(m)] \geq U_{s} \\
& \text { Constraints }(1),(2),(4) \text { and (5). }
\end{aligned}
$$

Since the throughput-energy curve is a non-decreasing function (Property 1) and that we have $f\left(P_{s}\right)=f\left(P_{\max }\right)=U_{s}$, the throughput-energy curve must be flat between $\left[P_{s}, P_{\text {max }}\right]$. Since $P_{s}$ is the minimum energy that achieves $U_{s}$, based on Property 1 , we have $f(P)<U_{s}$ for $P<P_{s}$.

The following property says that the segment of the optimal throughput-energy curve is strictly increasing for $P \in\left[0, P_{s}\right]$ (see Fig. 1).

Property 4: $f(P)$ is a strictly increasing function for $P \in$ $\left[0, P_{s}\right]$.

Proof: Our proof is based on contradiction. Suppose $f(P)$ is not strictly increasing within $\left[0, P_{s}\right]$. Since $f(P)$ is nondecreasing (Property 1), there must exist $0<P_{1}<$ $P_{2}<P_{s}$ such that $f\left(P_{1}\right)=f\left(P_{2}\right)<f\left(P_{s}\right)$. We can express $P_{2}$ as a linear combination of $P_{1}$ and $P_{s}$ as follows: $P_{2}=\lambda P_{1}+(1-\lambda) P_{s}$, where $0<\lambda<1$. Then, we have

$$
\begin{aligned}
\lambda f\left(P_{1}\right)+(1-\lambda) f\left(P_{s}\right) & >\lambda f\left(P_{2}\right)+(1-\lambda) f\left(P_{2}\right) \\
& =f\left(P_{2}\right) \\
& =f\left[\lambda P_{1}+(1-\lambda) P_{s}\right],
\end{aligned}
$$

where the first inequality holds since $f\left(P_{1}\right)=f\left(P_{2}\right)<f\left(P_{s}\right)$ and the third inequality holds since $P_{2}=\lambda P_{1}+(1-\lambda) P_{s}$. But this contradicts to the fact that $f(P)$ is a concave function.

Recall that all the weakly Pareto optimal points of MOPT coincide with the optimal throughput-energy curve $f(P)$ over $P \in\left[0, P_{\max }\right]$. It is easy to see that the points on $f(P)$ over $P \in\left[0, P_{s}\right]$ are Pareto optimal points (while those on $f(P)$ over $P \in\left(P_{s}, P_{\max }\right]$ are only weakly Pareto optimal points).

Although we have successfully analyzed some properties of the optimal throughput-energy curve, it remains difficult to characterize the entire curve for a given throughput utility function. A naive approach to approximate the curve is as follows. We can discretize the energy interval $\left[0, P_{s}\right]$ into a 
large number of equally spaced intervals. For each energy consumption value, $P_{i}$, we can compute its corresponding throughput value $f\left(P_{i}\right)$ by solving $\operatorname{OPT}\left(P_{i}\right)$. So we obtain a point $\left(P_{i}, f\left(P_{i}\right)\right)$ on the throughput-energy curve. Once we find all these points on the curve, we can connect them via linear segments. This will give us an approximate throughputenergy curve. Although this approach is simple and straightforward, it does not offer any performance guarantee of the curve.

In contrast, the goal of this paper is to characterize the curve with performance guarantee. In the following two sections, we consider two classes of throughput utility functions: the linear case and the non-linear case. In the linear case, we are able to characterize the optimal curve exactly by exploiting some special structures of linear program; for the nonlinear case, we develop a novel technique to approximate the curve with $(1-\varepsilon)$-optimal performance guarantee, where $\varepsilon$ is an arbitrary small error reflecting our desired accuracy.

\section{CASE 1: Linear Throughrut Function}

In this section, we consider the case where the throughput utility function is linear with respect to $r(m), m \in \mathcal{M}$. That is, $U=\sum_{m \in \mathcal{M}} w(m) r(m)$, where $w(m)$ is a constant and can be considered as the weight for session $m \in \mathcal{M}$. In this case, our OPT $(P)$ becomes the following LP.

$$
\begin{aligned}
\mathrm{LP}(P) \quad \max & U=\sum_{m \in \mathcal{M}} w(m) r(m) \\
\text { s.t. } & \text { All constraints in OPT(P) } \\
& r(m), r_{l}(m) \geq 0,0 \leq \alpha_{l} \leq 1 .
\end{aligned}
$$

Instead of obtaining the $f(P)$ curve by solving $\operatorname{LP}(P)$ for all possible $P \in\left[0, P_{\max }\right]$, which is unpractical, we will exploit the special structure of LP and obtain the $f(P)$ curve by solving a finite number of LPs. In particular, since LP $(P)$ is parametric linear program with respect to $P$, we propose to employ the so-called parametric analysis (PA) technique [3, Chapter 6.8 ] to obtain $f(P)$ curve efficiently.

\section{A. Finding $f(P)$ Curve via Parametric Analysis}

The main idea of PA is to investigate how a perturbation on parameter $P$ will affect the optimality of $\operatorname{LP}(P)$. For a given value of $P$, the current optimal basis of $\operatorname{LP}(P)$ could still be optimal when there is a perturbation on $P$. Thus, the interval $\left[0, P_{s}\right]$ can be partitioned into consecutive small intervals, each corresponding to a different optimal basis. Within each small interval, the optimal basis to $\operatorname{LP}(P)$ is the same even $P$ varies. Further, we will show that $f(P)$ is linear within each small interval.

Partition $\left[0, P_{s}\right]$ into Smaller Intervals. We now show how to partition interval $\left[0, P_{s}\right]$ into small intervals. Rewrite $\mathrm{LP}(P)$ in the standard form Max $\mathbf{c x}$, s.t. $\mathbf{A x}=\mathbf{b}$ and $\mathbf{x} \geq \mathbf{0}$. Here we use boldface to denote vectors and matrices. For a fixed value of $P$, this LP can be solved via standard technique in polynomial time [3, Chapter 8.4]. Corresponding to this $P$, suppose that we have an optimal basic matrix $\mathbf{B}$ and the

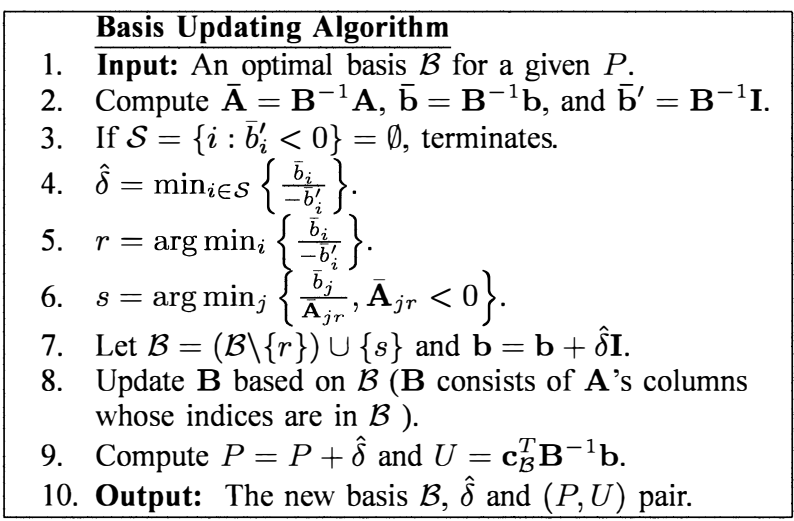

Fig. 2. The basis updating algorithm.

nonbasic matrix is $\mathbf{Q}$. Assume that the optimal solution to $\operatorname{LP}(P)$ is $\left(\mathbf{x}_{\mathcal{B}}, \mathbf{x}_{\mathcal{Q}}\right)$, where $\mathbf{x}_{\mathcal{B}}$ and $\mathbf{x}_{\mathcal{Q}}$ denote the values of basic and non-basic variables, respectively; $\mathcal{B}$ and $\mathcal{Q}$ denote the sets of basic and non-basic variables, respectively. Denote $\mathbf{c}_{\mathcal{B}}$ and $\mathbf{c}_{\mathcal{Q}}$ the objective function coefficient vectors of throughput utility $U$ for the basic and non-basic variables, respectively. Then we can write the corresponding canonical equations as follows:

$$
\begin{aligned}
U+\left(\mathbf{c}_{\mathcal{B}}^{T} \mathbf{B}^{-1} \mathbf{Q}-\mathbf{c}_{\mathcal{Q}}\right) \mathbf{x}_{\mathcal{Q}} & =\mathbf{c}_{\mathcal{B}}^{T} \mathbf{B}^{-1} \mathbf{b} \\
\mathbf{x}_{\mathcal{B}}+\mathbf{B}^{-1} \mathbf{Q} \mathbf{x}_{\mathcal{Q}} & =\mathbf{B}^{-1} \mathbf{b}
\end{aligned}
$$

Suppose we do a perturbation on parameter $P$, i.e., we change $P$ to $P+\delta$, then vector $\mathbf{b}$ becomes $\mathbf{b}+(\delta, 0, \ldots, 0)^{T}$. Then, the only change due to this perturbation is that $\mathbf{B}^{-1} \mathbf{b}$ will be replaced by $\mathbf{B}^{-1}(\mathbf{b}+\delta \mathbf{I})$, where vector $\mathbf{I}$ has a single 1 on the first element and zero on all the others. Note that $\mathbf{B}^{-1}(\mathbf{b}+\delta \mathbf{I})$ is a basic feasible solution (BFS). As long as $\mathbf{B}^{-1}(\mathbf{b}+\delta \mathbf{I})$ is nonnegative, the current basis remains optimal. The optimal basis will change only when one of the elements in $\mathbf{B}^{-1}(\mathbf{b}+\delta \mathbf{I})$ becomes negative. The value of $\delta$ at which this change occurs can be determined as follows. Denote $\overline{\mathbf{b}}=\mathbf{B}^{-1} \mathbf{b}$ and $\overline{\mathbf{b}}^{\prime}=\mathbf{B}^{-1} \mathbf{I}$, and let $\mathcal{S}=\left\{i: \bar{b}_{i}^{\prime}<0\right\}$, where $\bar{b}_{i}^{\prime}$ is the $i$-th element in vector $\overline{\mathbf{b}}^{\prime}$. If $\mathcal{S}=\emptyset$, then the current basis is optimal for all values of $\delta \geq 0$ since all elements in vector $\mathbf{B}^{-1}(\mathbf{b}+\delta \mathbf{I})$ are nonnegative. Otherwise, let

$$
\hat{\delta}=\min _{i \in \mathcal{S}}\left\{\frac{\bar{b}_{i}}{-\bar{b}_{i}^{\prime}}\right\} .
$$

For $\delta \in[0, \hat{\delta}]$, the current basis $\mathbf{B}$ remains optimal and its corresponding $\mathrm{BFS}$ is $\mathbf{x}_{\mathcal{B}}=\mathbf{B}^{-1}(\mathbf{b}+\delta \mathbf{I})$. When $\delta>\delta^{\prime}$, the basis $\mathbf{B}$ is no longer optimal. Thus, we need to choose the variable $x_{r}$ to leave the basis, where the minimum in (7) is attained for $i=r$. The entering variable $x_{s}$ is chosen by the dual simplex method rule [3, Chapter 6.8]. Based on the new optimal basis obtained after the pivot, we can update the corresponding canonical equations and get a $(P, U)$ pair, which is an endpoint of the linear segment of $f(P)$.

Figure 2 lists the steps to obtain a new optimal basis for a given optimal basis $\mathcal{B}$. Thus, starting from $P=0$, we can use this algorithm iteratively to find different bases until we reach 
TABLE I

SOURCE AND DESTINATION NODES OF EACH SESSION.

\begin{tabular}{|c|c|c|}
\hline Session $m$ & Source Node & Destination Node \\
\hline 1 & N19 & N15 \\
\hline 2 & N2 & N10 \\
\hline 3 & N8 & N12 \\
\hline 4 & N9 & N3 \\
\hline 5 & N5 & N1 \\
\hline 6 & N1 & N12 \\
\hline 7 & N4 & N11 \\
\hline 8 & N6 & N10 \\
\hline 9 & N16 & N6 \\
\hline 10 & N3 & N5 \\
\hline
\end{tabular}

$P_{s}$. The series of $\hat{\delta}$ for these bases will partition $\left[0, P_{s}\right]$ into small intervals.

The complexity of the basis updating algorithm can be analyzed as follows. The dominant computational complexity occurs in step 2: $\overline{\mathbf{A}}=\mathbf{B}^{-1} \mathbf{A}$. Note that our linear programming $\mathrm{LP}(P)$ has $Z=(1+2|\mathcal{L}|+|\mathcal{N}|-|\mathcal{M}|)$ constraints and $V=(|\mathcal{L}| \cdot|\mathcal{M}|+2|\mathcal{L}|+|\mathcal{M}|)$ variables. Since $\overline{\mathbf{A}}=\mathbf{B}^{-1} \mathbf{A}$ involves matrix multiplication of a $Z \times Z$ matrix and a $Z \times V$ matrix, its complexity is $O\left(Z^{2} V\right)=$ $O\left(|\mathcal{L}|^{3}|\mathcal{M}|+|\mathcal{N}|^{2}|\mathcal{L}||\mathcal{M}|+|\mathcal{N}||\mathcal{L}|^{2}|\mathcal{M}|\right)$.

Linearity of Each Small Interval. For each small interval with an optimal basis, we now show that $f(P)$ is linear. Suppose interval $\left[0, P_{s}\right]$ is divided into $K$ small intervals $\left[P_{i}, P_{i+1}\right], i=1, \ldots, K$, where $P_{1}=0, P_{K+1}=P_{s}$, and the optimal basis for small $\left[P_{i}, P_{i+1}\right]$ is $\mathbf{B}_{i}$. Then, for an optimal basis $\mathbf{B}_{i}$ within a particular small interval $\left[P_{i}, P_{i+1}\right]$, the objective value of throughput $f(P), P_{i} \leq P \leq P_{i+1}$ can be computed as follows.

$$
f(P)=\mathbf{c}_{\mathcal{B}_{i}}^{T} \mathbf{B}_{i}^{-1}(\mathbf{b}+\delta \mathbf{I}),
$$

where $\delta=P-P_{i}$. Substituting $\delta=P-P_{i}$ into (8), we have

$$
f(P)=\mathbf{c}_{\mathcal{B}_{i}}^{T} \mathbf{B}_{i}^{-1}\left[\mathbf{b}+\left(P-P_{i}\right) \mathbf{I}\right] .
$$

In (9), since $\mathbf{c}_{\mathcal{B}_{i}}^{T}, \mathbf{B}_{i}^{-1}, \mathbf{b}, \mathbf{I}$ and $P_{i}$ are constants, and $P$ is the only variable, we conclude that $f(P)$ is a linear function of $P$ for $P_{i} \leq P \leq P_{i+1}, i=1, \ldots, K$. We formally state this result in the following lemma.

Lemma 3: For the linear case, the optimal throughputenergy curve $f(P)$ is piece-wise linear within $\left[0, P_{s}\right]$.

Recall that by executing the basis updating algorithm sequentially, we also obtain a series of $(P, U)$ pair, each corresponding to an optimal basis. Since $f(P)$ is a piece-wise linear line with each linear segment determined by an optimal basis, the series of $(P, U)$ pairs are the endpoints of these linear segments. Then, by connecting these endpoints consecutively, we are able to obtain the entire optimal throughput-energy curve $f(P)$.

\section{B. A Numerical Example}

In the following, we present some pertinent numerical results to demonstrate our theoretical findings. We first describe our simulation settings. As shown in Fig. 3, we consider a randomly generated multi-hop wireless network with 20 nodes,

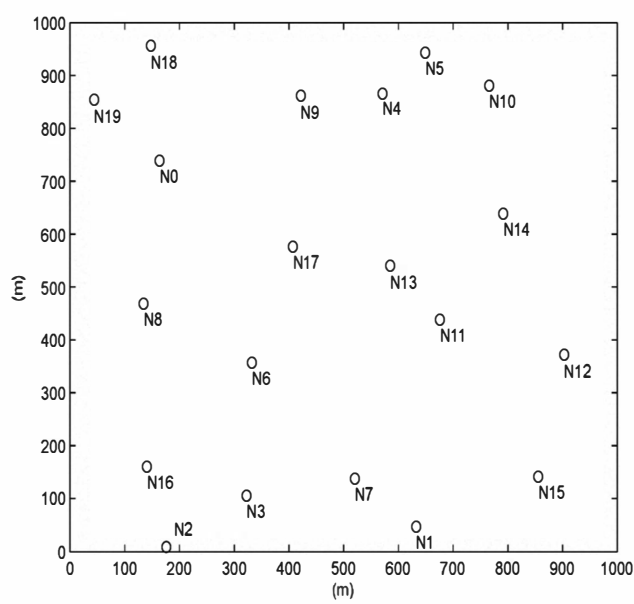

Fig. 3. Topology for a 20-node network.

which are distributed in a square region of $1000 \mathrm{~m} \times 1000 \mathrm{~m}$. The transmission power and reception power for each node are set to $P_{T}=1 \mathrm{~W}$ and $P_{R}=0.2 \mathrm{~W}$. The channel bandwidth on each link is $B=1 \mathrm{MHz}$. We use a simplified channel gain model $g_{l}=d_{l}^{-\gamma}$, where $d_{l}$ is the distance between the transmitter and receiver of link $l$ and $\gamma$ is the path loss index. We set $\gamma=3$. There are ten user sessions in the network and Table I specifies the source and destination nodes of each session. For the weight $w(m)$ of each session $m \in \mathcal{M}$, we consider two scenarios: (i) equal weight, e.g., $w(m)=1$ for all $m \in \mathcal{M}$; and (ii) random weight for each session.

The top curve in Fig. 4(a) shows the throughput-energy curve when each session has an equal weight of 1 . At the saturation point, we have $P_{s}=50.12$ and $U_{s}=120.02$. This curve is obtained by using PA method, which gives us 33 endpoints that interconnect the piece-wise linear segments of $f(P)$. Note that for each endpoint, our algorithm also provides its corresponding optimal solution of multi-hop routing variables $r(m), r_{l}(m)$ and each link's active time $\alpha_{l}$. For comparison, the bottom curve in Fig. 4(a) shows the throughput-energy curve under the popular minimum energy routing scheme [33], where each session chooses the path consuming the minimum energy. The minimum energy path for a session can be computed by using the well-know shortest path algorithms, (e.g., Dijkstra's algorithm or Bellman-Ford algorithm [11]), where the link cost on link $l$ is set to the total energy consumed to send one bit from the transmitter to the receiver, i.e., $C_{l} /\left(P_{T}+P_{R}\right)$. The large gap between throughput utility of the two curves shows that minimumenergy routing is far from optimal in terms of throughputenergy curve. This result affirms the importance of employing multicriteria formulation as we have done in this paper.

Figure 4(b) shows the results for the case when the weight of each session is randomly chosen. The randomly generated weights for the ten sessions are $0.8147,0.9058,0.1270$, $0.9134,0.6324,0.0975,0.2785,0.5469,0.1270$ and 0.9134 , respectively. Again, the throughput-energy curve is of the same form as that in Fig. 4(a), as expected. At the saturation point, we have $P_{s}=60.43$ and $U_{s}=72.11$. The bottom curve in 


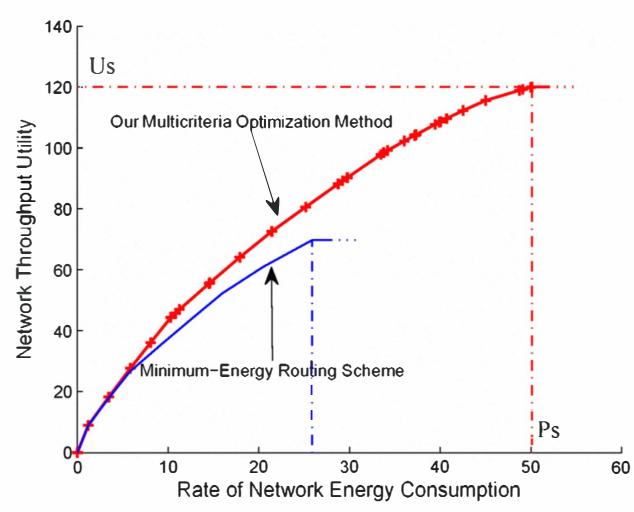

(a) Equal weight

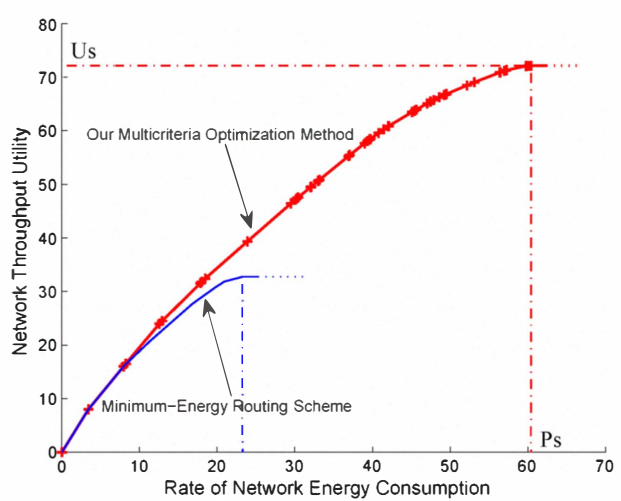

(b) Random weight

Fig. 4. The throughput-energy curves for a 20-node example.

Fig. 4(b) shows the throughput-energy curve under minimum energy routing, which is far from optimal.

\section{Case 2: Nonlinear Throughput Function}

In this section, we consider the case where the throughput utility function $h(\cdot)$ is a concave, but nonlinear function of $r(m), m \in \mathcal{M}$. In particular, we consider $h[r(m)]=$ $\ln [r(m)], m \in \mathcal{M}$, which is called proportional fairness in [20]. In this case, for a given $P, \operatorname{OPT}(P)$ is a convex, nonlinear program. Although convex program OPT $(P)$ can be solved efficiently for one given $P$, it is impractical to solve infinite number of such convex problems when $P$ varies from 0 to $P_{\max }$. Further, due to nonlinearity, we cannot take advantage of such technique as PA to compute the exact optimal throughput-energy curve efficiently.

Instead of finding the exact optimal throughput-energy curve, we propose a piece-wise linear approximation for this curve, where the approximation is guaranteed to be within $(1-\varepsilon)$-optimal, with $\varepsilon$ being an arbitrary small number. Note that for a given $P$, we can always find a corresponding $U$ on the optimal throughput-energy curve by solving a convex program (see Lemma 1). So the question becomes how to choose a set of such points and connect them with piece-wise linear segments such that this piece-wise linear approximation is no more than $\varepsilon$ (in percentile) from the unknown optimal throughput-energy curve.

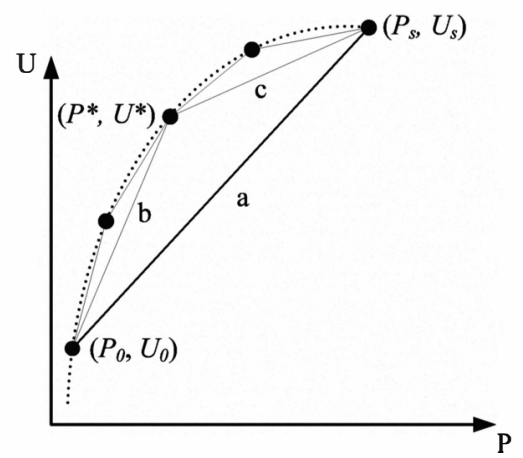

Fig. 5. An illustration of our piece-wise linear approximation method

First, we identify the two endpoints on the optimal throughput-energy curve that we want to approximate. On the left side, since the throughput utility is a $\ln (\cdot)$ function, it is negative when $P$ is small. Assuming we are only interested in the optimal throughput-energy curve when $f(P) \geq 0$, we will pick a $P$, denoted as $P_{0}$, such that $U_{0}=f\left(P_{0}\right)$ is just above zero. ${ }^{1}$ On the right side, recall that the optimal throughputenergy curve $f(P)$ is flat from $P=P_{s}$ to $P=P_{\max }$ So we can choose the saturation point $\left(P_{s}, U_{s}\right)$ (see Section III on how to obtain it) as our right endpoint.

With our two endpoints on the optimal throughput-energy curve being $\left(P_{0}, U_{0}\right)$ and $\left(P_{s}, U_{s}\right)$, our approximation method works as follows (see Fig. 5). We connect points $\left(P_{0}, U_{0}\right)$ and $\left(P_{s}, U_{s}\right)$ with a linear segment $a$ and consider it as our first approximation of the optimal throughput-energy curve. To examine if linear segment $a$ is accurate enough, we compute an error upper bound $\sigma$ of this approximation (in percentile). This is not trivial and will be shown in Lemma 4. If $\sigma \leq \varepsilon$, then our linear approximation is considered accurate enough and we are done. Otherwise, we will find a point $\left(P^{*}, U^{*}\right)$ on the optimal throughput-energy curve and use two linear segments $b$ and $c$ as a better approximation. Again, finding this point $\left(P^{*}, U^{*}\right)$ is not trivial (as the complete optimal throughputenergy curve is unknown) and will be explained shortly. Now the same process continues on linear segments $b$ and $c$. The process continues until $\sigma \leq \varepsilon$ for every linear segment of the piece-wise linear approximation curve.

We first show how to compute $\left(P^{*}, U^{*}\right)$, since we need $\left(P^{*}, U^{*}\right)$ when computing $\sigma$.

Finding $\left(P^{*}, U^{*}\right)$. Point $\left(P^{*}, U^{*}\right)$ has the maximum approximation error when we use a line segment to approximate a segment of the optimal throughput-energy curve (see Fig. 6).

Suppose that $\left(P_{1}, U_{1}\right)$ and $\left(P_{2}, U_{2}\right)$ are two endpoints of a line segment, which we denote as $\tilde{f}(P)$. Then this line segment $\tilde{f}(P)$ can be characterized as $\tilde{f}(P)=U_{1}+$ $\frac{U_{2}-U_{1}}{P_{2}-P_{1}}\left(P-P_{1}\right)$. Although the optimal throughput-energy curve $f(P)$ is unknown, we imagine that we move line $\tilde{f}(P)$ upward until it is tangential to the curve. Denote this tangential point as $\left(P^{*}, U^{*}\right)$, which is the point having the maximum absolute (rather than percentile) approxima-

\footnotetext{
${ }^{1}$ Note that $f\left(P_{0}\right)=0$ cannot be our left endpoint due to the singularity it presents when we compute the approximation error (in percentile)
} 


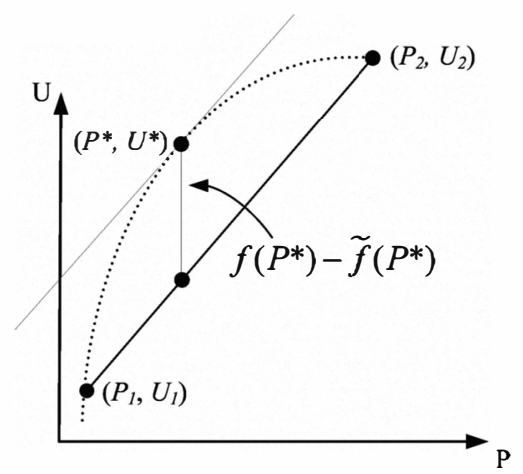

Fig. 6. An illustration showing how to obtain the tangential point and maximum approximation error on one linear segment.

tion error if we were to use $\tilde{f}(P)$ to approximate $f(P)$. Then, we have $f\left(P^{*}\right)-\tilde{f}\left(P^{*}\right)=\max \{f(P)-\tilde{f}(P)\}=$ $\max \left\{\sum_{m \in \mathcal{M}} h[r(m)]-\left[U_{1}+\frac{U_{2}-U_{1}}{P_{2}-P_{1}}\left(P-P_{1}\right)\right]\right\}$. Therefore, the tangential point $\left(P^{*}, U^{*}\right)$ can be found by solving the following optimization problem.

$$
\begin{array}{ll}
\max & \sum_{m \in \mathcal{M}} h[r(m)]-\left[U_{1}+\frac{U_{2}-U_{1}}{P_{2}-P_{1}}\left(P-P_{1}\right)\right] \\
\text { s.t. } & \sum_{l \in \mathcal{L}} \alpha_{l}\left(P_{T}+P_{R}\right)-P=0
\end{array}
$$

All constraints in MOPT,

where $P$ is also a variable. Note that the above optimization problem is a convex problem, which can be solved efficiently by using subgradient method [4, Chapter 8.9].

Finding $\sigma$. After obtaining the tangential point $\left(P^{*}, U^{*}\right)$, we can calculate an upper bound $\sigma$ of the approximation error (in percentile) with the following lemma.

Lemma 4: An error upper bound of using $\tilde{f}(P)$ as the approximation of $f(P), P_{1} \leq P \leq P_{2}$, is $\sigma=1 /\left[1+\frac{U_{1}}{U^{*}-\hat{f}\left(P^{*}\right)}\right]$.

Proof: Referring to Fig. 6, for any point $(P, f(P))$ within $\left[P_{1}, P_{2}\right]$, the approximation error (in percentile) is

$$
\frac{f(P)-\tilde{f}(P)}{f(P)}=\frac{f(P)-\tilde{f}(P)}{f(P)-\tilde{f}(P)+\tilde{f}(P)}=\frac{1}{1+\frac{\tilde{f}(P)}{f(P)-\tilde{f}(P)}} .
$$

Since $\tilde{f}(P) \geq f\left(P_{1}\right)=U_{1}$ and $f(P)-\tilde{f}(P) \leq U^{*}-\tilde{f}\left(P^{*}\right)$, we have

$$
\frac{f(P)-\tilde{f}(P)}{f(P)}=\frac{1}{1+\frac{\tilde{f}(P)}{f(P)-\tilde{f}(P)}} \leq \frac{1}{1+\frac{U_{1}}{U^{*}-\tilde{f}\left(P^{*}\right)}}=\sigma .
$$

Now given that we can compute $\sigma$ at each iteration and our process stops when $\sigma \leq \varepsilon$ for each segment, it is not hard to see that our piece-wise linear approximation can guarantee $(1-\varepsilon)$-optimal. We state this result in the following theorem.

Theorem 1: For any small $\varepsilon>0$, the proposed piece-wise linear approximation method can approximate the optimal throughput-energy curve $f(P)$ with $(1-\varepsilon)$-optimal.

A Numerical Example. We now use a numerical example to illustrate the optimal throughput-energy curve when the

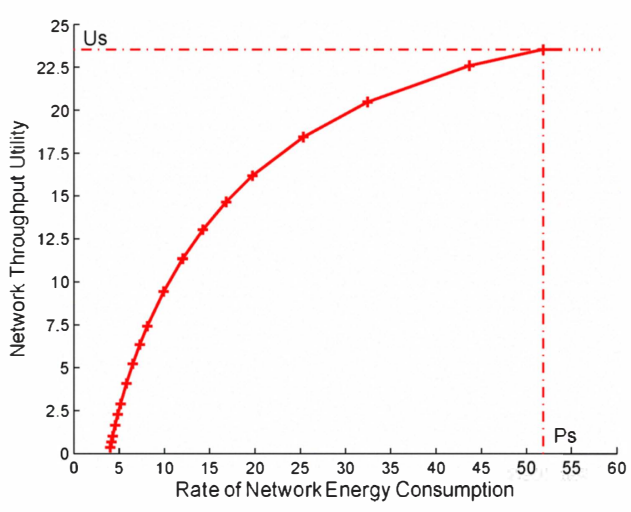

Fig. 7. A $(1-\varepsilon)$-optimal throughput-energy curve for the nonlinear case. $\varepsilon=1 \%$.

throughput utility function $h[r(m)]=\ln [r(m)]$. We use the same setting as that of the numerical example shown in Section IV. The network topology is shown in Fig. 3. We first determine the suturation point $\left(P_{s}, U_{s}\right)$ based on the approach presented in Section III. On the left, we find $P(3.86)=0$. So we choose $P_{0}=4>3.86$ and find its corresponding throughput utility $f\left(P_{0}\right)=0.35$. On the right, we find the saturation point $\left(P_{s}, U_{s}\right)=(51.83,23.54)$. Now we will approximate the optimal throughput-energy curve $f(P)$ for $P \in[4.00,51.83]$. Suppose we set the target approximation error $\varepsilon=1 \%$, i.e., we are pursuing a $99 \%$-optimal piecewise linear approximation. Using the method described in this section, we obtain 18 piece-wise linear segments shown in Fig. 7, corresponding to linear connection of 19 points on the optimal throughput-energy curve.

\section{CONCLUSION}

In this paper, we explored the relationship between two key performance metrics of a multi-hop wireless network: network throughput and energy consumption. By casting the problem into a multicriteria optimization, we showed that the solution to this problem is equivalent to finding the so-called optimal throughput-energy curve of the network. Subsequently, we presented a number of important properties associated with the optimal throughput-energy curve. As for case study, we considered both the linear and nonlinear throughput functions. For the linear case, we were able to characterize the optimal throughput-energy curve precisely via parametric analysis. For the nonlinear case, we proposed a piece-wise linear approximation that can guarantee $(1-\varepsilon)$-optimal.

In theory, the characterization of optimal throughput-energy curve is a significant advance over the state-of-the-art, which is limited to either maximizing throughput under some energy constraint or minimizing energy consumption while satisfying some throughput requirement, with either being able to offer only a single point on the optimal throughput-energy curve. In practice, the optimal throughput-energy curve is very useful for a network designer or operator, as it offers a holistic view on the two performance metrics. A network designer/operator 
can achieve a desired trade-off between the two metrics depending on the specific network application scenarios.

\section{ACKNOWLEDGMENTS}

The work of Y.T. Hou, Y. Shi, and C. Jiang was supported in part by NSF under Grant ECCS-0925719. The work of S. Kompella was supported in part by the ONR.

\section{REFERENCES}

[1] M. Al-Ayyoub and H. Gupta, "Joint routing, channel assignment, and scheduling for throughput maximization in general interference models," IEEE Trans. on Mobile Computing, vol. 9, no. 4, pp. 553-565, April 2010.

[2] M. Alicherry, R. Bhatia, and L. Li, "Joint channel assignment and routing for throughput optimization in multi-radio wireless mesh networks," in Proc. ACM MobiCom, pp. 58-72, Cologne, Germany, Aug. 2005.

[3] M.S. Bazaraa, J.J. Jarvis, and H.D. Sherali, Linear Programming and Network Flows, 3rd edition, John Wiley \& Sons Inc., Hoboken, New Jersey, 2005

[4] M.S. Bazaraa, H.D. Sherali, and C.M. Shetty, Nonlinear Programming: Theory and Algorithms, 3rd edition, John Wiley \& Sons Inc., Hoboken, New Jersey, 2006.

[5] R. Bhatia and M. Kodialam, "On power efficient communication over multi-hop wireless networks: Joint routing, scheduling and power control," in Proc. IEEE INFOCOM, pp. 1457-1466, Hong Kong, China, March 2004

[6] S. Chachulski, M. Jennings, S. Katti and D. Katabi, " Trading structure for randomness in wireless opportunistic routing," in Proc. ACM SIGCOMM, pp. 169-180, Kyoto, Japan, Aug. 2007.

[7] D. Chafekar, V.S.A. Kumar, M. Marathe, S. Parthasarathy, and A. Srinivasan, "Approximation algorithms for computing capacity of wireless networks with SINR constraints," in Proc. IEEE INFOCOM, pp. 11661174, Phoenix, AZ, April 2008.

[8] P. Chaporkar, K. Kar, and S. Sarkar, "Throughput guarantees through maximal scheduling in wireless networks," in Proc. 43rd Ann. Allerton Conf. Commun., Control and Comput., pp. 1557-1567, Monticello, IL, Sep. 2005.

[9] M. Chiang, "Balancing transport and physical layers in wireless multihop networks: Jointly optimal congestion control and power control," IEEE Journal on Selected Areas in Communications, vol. 23, no. 1, pp. 1166-1174, Jan. 2005.

[10] S. Cui, A.J. Goldsmith, and A. Bahai, "Energy-constrained modulation optimization," IEEE Trans. on Wireless Communications, vol. 4, no. 5, pp. 2349-2360, Sep. 2005.

[11] T.J. Cormen, C.E. Leiserson, R.L. Rivest, and C. Stein, Introduction to Algorithms, Second Edition, The MIT Press, 2001.

[12] L. Chen, S.H. Low, M. Chiang, and J.C. Doyle, "Cross-layer congestion control, routing and scheduling design in ad hoc wireless networks," in Proc. IEEE INFOCOM, 13 pages, Barcelona, Spain, April 2006.

[13] D.S.J. De Couto, D. Aguayo, J. Bicket, and R. Morris, "A highthroughput path metric for multi-hop wireless routing," Springer Wireless Networks, vol. 11, no. 4, pp. 419-434, July 2005.

[14] R.L. Cruz and A.V. Santhanam, "Optimal routing, link scheduling, and power control in multi-hop wireless networks," in Proc. IEEE INFOCOM, pp. 702-711, San Francisco, CA, March 30-April 3, 2003.

[15] Q. Dong, S. Banerjee, M. Adler, and A. Misra, "Minimum energy reliable paths using unreliable wireless links," in Proc. ACM MobiHoc, pp. 449-459, Urbana-Champaign, IL, May 2005.

[16] M. Ehrgott, Multicriteria Optimization, Springer-Verlag New York, 2005.

[17] A. El Gamal, C. Nair, B. Prabhakar, E. Uysal-Biyikoglu, and S. Zahedi, "Energy-efficient scheduling of packet transmissions over wireless networks," in Proc. IEEE INFOCOM, pp. 1773-1782, New York, NY, June 2002.

[18] W.R. Heinzelman, A. Chandrakasan, and H. Balakrishnan, "Energyefficient communication protocol for wireless microsensor networks," in Proc. of the 33rd Hawaii International Conference on System Sciences, pp. 3005-3014, Maui, Hawaii, Jan. 2000.
[19] C. Jiang, Y. Shi, Y.T. Hou, and S. Kompella, "On optimal throughput-energy curve for multi-hop wireless networks," Technical Report, the Bradley Department of Electrical and Computer Engineering, Virginia Tech, Blacksburg, VA, July 2010. Available at http://filebox.vt.edu/users/cmjiang/Jiang10TR.pdf.

[20] F.P. Kelly, A. Maulloo, and D. Tan, "Rate control in communication networks: shadow prices, proportional fairness and stability," Journal of the Operational Research Society, vol. 49, no. 3, pp. 237-252, March 1998.

[21] Y. Kim, H. Shin, and H. Cha, "Y-MAC: An energy-efficient multichannel MAC protocol for dense wireless sensor networks," in Proc. of the 7th International Conference on Information Processing in Sensor Networks, pp. 53-63, St. Louis, Missouri, USA, April 2008.

[22] M. Kodialam and T. Nandagopal, "Characterizing achievable rates in multi-hop wireless mesh networks with orthogonal channels," IEEE/ACM Trans. on Networking, vol. 13, no. 4, pp. 868-880, Aug. 2005.

[23] S. Kwon and N.B. Shroff, "Unified energy-efficient routing for multi-hop wireless networks," in Proc. IEEE INFOCOM, pp. 430-438, Phoenix, AZ, April 2008

[24] W. Li and H. Dai, "Optimal throughput and energy efficiency for wireless sensor networks: Multiple access and multipacket reception," EURASIP Journal on Wireless Communications and Networking, vol. 5, issue 4, pp. 541-553, Sep. 2005.

[25] L. Lin, X. Lin, and N.B. Shroff, "Low-complexity and distributed energy minimization in multihop wireless networks," IEEE/ACM Trans. on Networking, vol. 18, no. 2, pp. 501-514, April 2010.

[26] X. Lin and N.B. Shroff, "The impact of imperfect scheduling on crosslayer congestion control in wireless networks," IEEE/ACM Trans. on Networking, vol. 14, no. 2, pp. 302-315, April 2006.

[27] X. Lin and S. Rasool, "Distributed and provably efficient algorithms for joint channel-assignment, scheduling, and routing in multichannel ad hoc wireless networks," IEEE/ACM Trans. on Networking, vol. 17 , no. 6, pp. 1874-1887, Dec. 2009.

[28] G. Lu, N. Sadagopan, B. Krishnamachari, and A. Goel, "Delay efficient sleep scheduling in wireless sensor networks," in Proc. of IEEE INFOCOM, pp. 2470-2481, Miami, FL, March 2005.

[29] I. Maric and R.D. Yates, "Cooperative multihop broadcast for wireless networks," IEEE Journal on Selected Areas in Communications, vol. 22, issue 6, pp. 1080-1088, Aug. 2004.

[30] H. Nama, M. Chiang, and N. Mandayam, "Utility-lifetime trade-off in self-regulating wireless sensor networks: A cross-layer design approach," in Proc. IEEE ICC, pp. 3511-3516, Istanbul, Turkey, June 2006.

[31] M.J. Neely, "Energy optimal control for time varying wireless networks," IEEE Trans. on Information Theory, vol. 52, no. 7, pp. 2915-2934, July 2006.

[32] M.J. Neely and R. Urgaonkar, "Optimal backpressure routing in wireless networks with multi-receiver diversity," Ad Hoc Networks (Elsevier), vol. 7, no. 5, pp. 862-881, July 2009.

[33] S. Singh, M. Woo, and C.S. Raghavendra, "Power-aware routing in mobile ad hoc networks," in Proc. ACM MobiCom, pp. 181-190, Dallas, Texas, Oct. 1998.

[34] Y. Sun, S. Du, O. Gurewitz, and D.B. Johnson, "DW-MAC: A low latency, energy efficient demand-wakeup MAC protocol for wireless sensor networks," in Proc. ACM MobiHoc, pp. 53-62, Hong Kong, China, May 2008

[35] A. Tarello, J. Sun, M. Zafer, and E. Modiano, "Minimum energy transmission scheduling subject to deadline constraints," Springer Wireless Networks, vol. 14, no. 5, pp. 633-645, 2008

[36] L. Tassiulas and A. Ephremides, "Stability properties of constrained queueing systems and scheduling policies for maximum throughput in multihop radio networks," IEEE Trans. on Automatic Control, vol. 37 , no. 12, pp. 1936-1948, Dec. 1992.

[37] T. Van Dam and K. Langendoen, "An adaptive energy-efficient MAC protocol for wireless sensor networks," in Proc. ACM SenSys, pp. 171180, Los Angeles, CA, Nov. 2003.

[38] X. Wu and R. Srikant, "Regulated maximal matching: A distributed scheduling algorithm for multi-hop wireless networks with nodeexclusive spectrum sharing," in Proc. IEEE CDC, pp. 5342-5347, Seville, Spain, Dec. 2005.

[39] W. Ye, J. Heidemann, and D. Estrin, "An energy-efficient MAC protocol for wireless sensor networks," in Proc. IEEE INFOCOM, pp. 15671576, New York, NY, June 2002. 